\title{
Simone Dietz
}

\section{Die Lüge von der »Auschwitzlüge - Wie weit reicht das Recht auf freie Meinungsäußerung?}

Jeder kennt das Gefühl der Ohnmacht und Wur, das sich einstellt, wenn man mit einer offensichelich falschen Bchauptung konfrontiert ist, die ihr Verfechter aller argumentativen Widerlegung zum Trotz aufrechterhält. Der schulterzuckende Abbruch des Gesprächs, die Hoffnung darauf, daß sich die Wahrheit auf Dauer selbst erweisen, oder daß eine offensichtlich falsche Behauptung bei anderen keine große Beachtung finden werde, kommen gegen die relativierende Macht solcher Opposition letztlich nicht an. Solange auch nur ein Mensch die Anerkennung der Wahrheir offentlich verweigert, ist der Zweifel daran in der Welt. Besondere Schärfe gewinnt ein solcher Konflikr, wenn mir der umstrittenen Behauptung die Identität von Personen auf dem Spiel steht. Denn dann geht es nicht mehr allein um das epistemische Problem der Anerkennung wahrer Behauptungen, sondern auch um das moralische Problem der Anerkennung bzw. Mißachtung persönlicher Identität.

Um einen solchen Fall handele es sich bei der Behauptung, im Konzcntrationslager von Auschwitz habe es keine Gaskammern und überhaupt habe es im "Dritten Reich" keine geziclte Massenvernichtung von Juden gegeben. ${ }^{1}$ Die Konfrontation mit den von Deutschen im Namen Deutschlands begangenen Verbrechen während der Zeit des Nationalsozialismus isc ein Thema, das wie kein anderes in Deutschland geeigner ist, um Gefühle der Ohnmacht, Enıpörung und Scham hervorzurufen. Insofern zielt das Bestreiten der systematischen Verfolgung und Ermordung der Juden auf ein Faktum von höchster moralischer und politischer Bedeutung, und es ist nicht verwunderlich, daß die Frage nach dem angemessenen politischen Umgang mit offenkundig falschen Behauptungen gerade an diesem Fall immer wieder neu diskutiert wird.

Die Frage der Strafbarkeit der öffentlichen Leugnung des Holocaust hatte den Deutschen Bundestag bereits $1959 / 60$, und zwischen $198 \mathrm{r}$ und $198 \mathrm{~s}$ beschäftigt.. ${ }^{2}$ Im Frühjahr 1994 entbrannte die Debatte anläßlich einiger umstrittener Gerichtsurteile von Neuem und führte schließlich zu cincr Gesctzesänderung, dic fast zehn Jahre zuvor bei cinem ähnlichen Vorschlag noch gescheitert war. Nach der neuen Fassung des $\$ 130$ Abs. 3 des Strafgesetzbuchs soll für Volksverherzung künftig ausdrücklich bestraft werden können, wwer eine unter der Herrschafe des Nationalsozialismus begangene Handlung der in $\$ 220$ a Abs. I bezeichneten Art« (Völkermord) »in einer

\footnotetext{
1 Der Ausdruck *Auschwitzlugew, ein Begriff der sogenannten Revisionisten für ihrc Behauptung, die Massenvernichtung der Juden in Auschwitz sei eine Luge, wird inzwischen auch umgekehrt verwendet, un die Luge zu bezeichnen, in Auschwizz habe es keme Massenvernichtung gegeben. Diese Neubesetzung des Begriffs verwischt allerdings die Grenze zwischen Leugnung und gezitler Difimierung. Eine nusfuhrliche Analyse der verschiedenen Strategien der Holocaust-Leugner hat Deborah E. Lipstadt geleistet in ihrem Buch: Betriff: Leugnen des Holocaust, Zurich 1994.

2 Zur Geschichte der Gesetzesinitiaciven und -anderungen bezuglich der Holocaust-Leugnung vgl. Sebastian Cobler, Das Gesetz gegen die "Auschwitz-Lüge๘, KJ 198, 159-170; Heribert Ostendorf, Die strafrechtliche Verfolgung der nAuschwitzlüge*, NJW 1989, 1062-1065; Horst Meier, D:2s Strafrecht gegen die $\times$ Auschruluzluge«, Merkur 1994, I $128-11$ j2.
} 
Weise, die geeignet ist, den öffentlichen Frieden zu stören, öffentlich oder in einer Versammlung billigt, leugnet oder verharmlost «. ${ }^{3}$ Die wesentlichen Unterschiede zwischen der neuen und der alten Gcsetzeslage bestehen darin, daß nun die Billigung, Leugnung und Verharmlosung des Holocaust ausdrücklich als Volksverhetzung gilt, und daß für ihre Bestrafung allein die mögliche Störung des öffentlichen Friedens, nicht aber wic bisher auch ein Angriff auf die Menschenwürde anderer nachgewiesen werden muß.

Auf beide Aspekte hatte sich zuvor auch die öffentliche Diskussion der erwähnten Gerichtsurteile konzentriert: die Begründung, mit der der Bundesgerichtshof ein Urteil des Landgerichts Mannheim autgehoben hatte, weil »das bloßc Bcstrcitcn der Gaskammermorde " noch keinen Angriff auf die Menschenwürde darstelle und damit "den Tatbestand der Volksverhetzung nicht erfüllt « ${ }^{4}$, wurde im In- und Ausland vielfach als Skandal aufgenommen. Wenn das Bestreiten des Völkermordes nicht als Volksverhetzung verurteilt werden könne, gebe es hier offenbar eine Gesetzeslücke, die geschlossen werden müsse, so wurde vielfach kritisiert, denn die Leugnung des Holocaust dürfe auf keinen Fall vom Grundrecht der Meinungsfreiheit geschützt sein. Es reiche auch nicht aus, daß entsprechende Äußerungen bereits indirekt unter Strafe stünden, indem man sie als persönliche Beleidigung (nach den $\$ \$$ r $8, \mathrm{ff}$. StGB) werte, weil dies eine unangemessenc "Privatisierung eines gesellschaftlichen Konflikts sei.s

Mit der Novellierung des Volksverhetzungsparagraphen wurde beabsichtigt, die einfache Leugnung des Holocaust direkr zur strafbaren Äußerung zu erklären, die cine verhetzende und Unfrieden stiftende Wirkung habe, und sie damit auch unabhängig von der mit ihr verbundenen indirckten Beleidigung der Opfer des Holocaust unter Strafe zu stellen. Gerade unter diesem Aspekt wird das neue Gesetz von anderer Seite aber kritisiert, indem geltend gemacht wird, daß das Strafreche die Rechte anderer, nicht aber historische Tatsachen zu schützen habe. Man dürfe nicht durch Sondergesetze bestimmte historische Tatsachen zur Wahrheit erklären. Ein solcher Versuch könne im Gegenteil gerade die zwingende Selbstverständlichkeit einer wahren Tatsache erschüttern, die sich immer im freien Streit der Meinungen gegen Zweifel durchsetzen können muß. ${ }^{6}$

3 Bundesgesetzblatt vom 4. 11. 1994, Nr.76;Z 5702 A, 3187 . Nach dem bisherigen $\$ 130$ konnte fur Volksverhetzung bestraft werden, wwer in einer Weise, die geeignet ist, den offentlichen Fneden zu storen, die Menschenwürde anderer dadurch angreift, daß er 1. zum Haß gegen Teile der Bevólkerung aufstachelt, 2. zu Gewalt- oder Willkirmaßnahmen gegen sie auffordert oder 3. sie beschimpft, böswillig verachtlich macht oder verleumdet . Die Rechtsprechung unterschied deshalb bisher zwischen der Leugnung des Holocaust als seinfacher Auschwitzluge $\alpha$, und der weitergehenden Behauptung von der Erfindung des Holocaust aus dem Motıv der Erpressung der Deutschen als squalifizierter Auschwitzluge . Nur die "qualifizierte Auschwitzluge galt bisher eindeutig als strafbar im Sinne des $\oint_{13} 0$ (Volksverhetzung) des StGB, insofern sıe Teile der Bevolkerung als angebliche Lugner und Ergresser ausdrucklieh verleumder (vgl. BGH-Beschluß vom 16. 11, 1993, NStZ 1994, 140).

4 BGH Urtel yom 15.3. 1994, NJW 5994, 1421. Das Landgerscht Mannheim hatte 1992 den NPD-Vorsitzenden G. Deckert wegen Volksverhetzung, ubler Nachrede, Verunglimpfung des Andenkens Verstorbener und Aufstachelung zum Rassenhaß verurteilt, weil er eine Vortragsveranstaltung mit dem Hauptrhema der Widerlegung des Holocaust durchgefuhrt hatte. Dieses Urteil wurde im Mirz 1994 vom Bundesgerichrshof wegen Begrundungsmangeln aufgehoben und an das Landgericht zurückverwsesen. Die Ncuverhandlung des Falles im Juni 1994 gab wegen einer gegenuber dem Angeklagten allzu verstandnisvollen Urteilsbegrundung wiederum Anlaß fur Emporung; auch dieses Urteil wurde vom Bundesgerichtshof wieder aufgehoben.

So dic Gruppe Bundnis 9o/Die Grunen im Bundestag in der Begrundung thres Antrags auf Gesetzesanderung (BT-Drs. 12/421, S. 3). Die Beleidigung von Teilen der Bevolkerung, die unter der NS-Herrschaft verfolgt wurden, gilt nach $\$ 194 \mathrm{StGB}$ immerhin als Offizialdelikt, das auch ohne Strafantrag von Amts wcgen verfolgt werden kann.

6 So u. a. G. Rocilecke: - Tatsachen konnen nur fur sich selbst sprechen und jeden Widerspruch ausschlieBen, wenn sie ohne Gutesiegel und ohne den Stempel sachlich richtıgs auf(reten. a FAZ, 18. 5.94, S. 13. Ábnlich argumentieren auch S. Cobler, a.a. O. und H. Meicr, a.a.O. (Anm. 2). 
An dieser Auseinandersetzung über die Strafbarkeit der Holocaust-Leugnung und die Bedeutung der Meinungsfreiheit hat sich das Bundesverfassungsgericht mit einer Stellungnahme beteiligt, die nicht nur politische, sondern auch philosophische Aufmerksamkeit verdient. Das Bundesverfassungsgericht vertritt darin die Auffassung, das Leugnen des Völkermords sei schon deshalb grundsätzlich nicht vom Grundrecht der Meinungsfreiheit geschützt, weil es sich dabei gar nicht um eine Meinung, sondern um eine falsche Tatsachenbehauptung handelt: "Meinungen sind durch die subjektive Beziehung des Einzelnen zum Inhalt seiner Aussage geprägt. Für sie ist das Element der Stellungnahme und des Dafürhaltens kennzeichnend. Insofern lassen sie sich auch nicht als wahr oder unwahr erweisen «. Bei Tatsachenbehauptungen stehe dagegen »die objektive Beziehung zwischen der Äußerung und der Realität im Vordergrund. Insofern sind sic auch einer Überprüfung auf ihren Wahrheitsgehalt zugänglich«. In diesem Sinne beziehe sich der entsprechendc Grundgesetzartikel (s, I, I) allein auf die Freiheit der Meinungsäußerung, Tatsachenbehauptungen hingegen seien nur geschützt, sofern sie Voraussetzung für die Bildung von Meinungen scien. Dies könne jedoch nicht für »die bewußt oder erwiesen unwahre Tatsachenbehauptung " - wie in Fall der Holocaust-Leugnung - geltend gemacht werden, da diese "zu der verfassungsrechtlich vorausgesetzten Meinungsbildung nichts beitragen könne«. Im übrigen sei auch die Schutzwürdigkeit von Meinungsäußerungen "vom Wahrheitsgehalt der ihnen zugrunde liegenden tatsächlichen Annahmen « abhängig. ${ }^{7} \mathrm{Das}$ Bundesverfassungsgericht schränkt also das Grundrecht auf freie Meinungsäußerung schon begrifflich, und nicht erst durch die mögliche Kollision mit anderen Rechten ein - und liefert damit eine Begründung, warum auch das Bestreiten historischer Tatsachen, und nicht nur der Angriff auf andere unter Strafe gestellt werden kann.

Ich möchte im folgenden das Verhältnis von Meinungsfreiheit und Tatsachenbehauptung am Beispiel der Holocaust-Leugnung erörtern, und dabci in zwei Schritten vorgehen: Zuerst werde ich mich mit der Argumentation des Bundesverfassungsgerichts auseinandersetzen, das Grundrecht auf freie Meinungsäußerung sei schon auf der begrifflichen Ebene durch die Unterscheidung zwischen Meinungsäußerungen und Tatsachenbchauptungen cingrenzbar. Ich möchte zeigen, warum diese Position mit einem liberalen Verständnis der Meinungsfreiheit unvereinbar ist. In einem zweiten Teil gehe ich darauf ein, inwiefern die Behauptung der "Auschwitzlüge eine Abwägung zwischen konfligierenden Rechten erfordert, und welche anderen Rechte hier gegenüber dcm Grundrecht der Meinungsfreiheit im einzelnen abgewogen werden müssen. In diesem Zusammenhang komme ich auf die Neufassung des Volksverhetzungsparagraphen zurück, die ich sowohl unter dem juristischen Aspekt der Abwägung zwischen konfligierenden Rechten, als auch unter dem politischen Aspekt des Umgangs mit der deutschen Geschichte für falsch halte.

Um Mißverständnisse zu vermeiden, möchte ich zuvor noch einmal klarstellen: mein Thema ist nicht die Behauptung der "Auschwitzlüge" selbst, sondern der politische und juristische Umgang mit solchen offenkundigen Falschbehauptungen. Es gcht mir nicht darum, den Holocaust-Leugnern ein Forum für ihre Falschbehauptungen zu erstreiten, sondern zu begründen, warum der juristische Weg, den das zitierte Bundesverfassungsgerichts-Urteil und die Novellierung des $₫ 130 \mathrm{StGB}$ gegenüber

7 t BvR 23/94, S. 9 ff. (NJW 1994, 1779). Es handelt sich um ein Urteil, mit dem das Bundesverfassungsgericht uber "die Zulassigkeit von Auflagen fur eine Versammlung, in der die Leugnung der Judenverfolgung im „Dritten Reich، zu enwarten ist * zu entscheiden hatte. Die Stadt Munchen hatte der NPD verordnet, sie musse auf ahrer Veranstaltung sicherstellen, daß die Judenverfolgung um Dritten Reich niche geleugnet oder bezweifelt werde. Das Bundesverfassungsgericht entsched, eme solche Auflage seı mit dem Grundrechi der Mcinungsfreiheit vereinbar. 
solchen Behauptungen einschlagen, letztlich nicht dem Interesse an historischer Tatsachenwahrheit dienen wird.

I

Ein juristisches Urteil mit philosophischen Begriffen zu überprüfen, und einem Gericht seinen naiven Wahrheitsbegriff nachzuwcisen, könnte leicht als wohlfeiles Unternehmen philosophischer Wichtigtuerei erscheinen. Gegen solches Abwinken spricht aber nicht nur der herausragende politische Stellenwert des Grundrechts auf Meinungsfreiheit und der Urteile des Bundesverfassungsgerichts im politischen System der Bundesrepublik. Es wäre auch ein Fehler, die hier zur Debatte stehende Begriffsdifferenzierung zwischen Tatsachenbehauptungen und Meinungsäußerungen als ad-hoc-Unterscheidung von untergeordneter Bedeutung einzuschätzen. In der juristischen Diskussion ist diese Unterscheidung durchaus cinschlägig, wenn auch nicht unumstritten - und von erheblicher Bedeutung für die Auslegung des Artikel s des Grundgesetzes. ${ }^{8}$ Auch in der philosophischen Diskussion hat sie Mitstreiter.

Die Unterscheidung zwischen Meinungen und Wahrheitserkenntnis steht als Differenzierung zwischen doxa und episteme in einer langen philosophischen Tradition, die allerdings auch eine Tradition des Streites um die Bedeutung beider Begriffe ist. Von der einen Seite wird der überzogene Anspruch auf ewige Wahrhciten kritisiert, den dogmatische Metaphysiker mit dem Begriff der episteme verbinden, auf der anderen Seite haftet dem Begriff der doxa der rclativistische Makel des bloß Subjektiven, Kontingenten und Vorläufigen an. Zwischen den extremen Positionen - der relativistischen, die bchauptet, es gebe überhaupt nur Meinungen und keine Wahrhciten, und der metaphysischen, die der Vernunfterkenntnis absolute Wahrheit zuschreibt - stehen Ansätze, die den Begriff der doxa von abwertenden Auffassungen rehabilitieren wollen, ohne andererseits auf den Begriff der episteme zu verzichten.

Im Kontext der politischen Theorie war es Hannah Arendt, die die doxa zum wichtigsten Grundbegriff des Politischen erhob, ohne den Begriff der absoluten Wahrheit preiszugeben. Gerade durch striktc Abgrenzung von der Wahrheitserkenntnis will sie den hohen Stellenwert der politischen Meinung unterstreichen. In ihrem Essay über »Wahrheit und Politik a aus dem Jahr $1967^{9}$ unterscheidet sie, ganz ähnlich wie das Bundesverfassungsgericht in dem zitierten Urteil von 1994, zwischen Meinungen und Tatsachen, und auch sie leitct aus dieser Unterscheidung eine grundsätzliche Beschränkung des politischen Rechts auf freie Mcinungsäußerung ab. Ich möchte

8 So beurteilen v. Mangoldt/Klein/Starck in ihrem Grundgesetz-Kommentar (GG, Art.s Rdnr. 16-20; Munchen 1985) die *Versuche, Tatsachenmitteilungen von Meınungen zu trennen « zwar außerst skeptisch, da wath die bloße Mitteilung von Tatsachen (...) allein durch die Auswahl bereits wertende Elemente: enthaite, schlıeßen sich aber dennoch der Auffassung an, ndic falsche Tatsachenmittellung sei nkein zu schurzendes Rechesgut, das vom Begriff der Meinung i.S. des Art. s erfaßt wird «, wobei sie immerhin einraumen: -Schwierigkeiten ergeben sich bei der Abgrenzung von wahr und falsch». Einen ahnlichen -Mittclweg - die strikte Unterscheidung zwischen Tatsachenbehauptungen und Meinungen zwar in Frage zu stellen, bei der Ausgrenzung falscher Tatsachenbehauptungen aber gleichwohl anzuwenden - schlagen auch Herzog (in: Maunz/Durig, GG, Arr. s Abs. I, II Rdnr. 50-55; 1992) und Jarass (in: Jarass/Pieroth, GG, Art. s Rdnr. 2; München 1992) em. Lediglich Wendt kritisiert die Ausgrenzung falscher Tatsachenmitreilung aus dem Schurzbereich des Art. s, I, i als wuberzogen objektivistisch-institutionelle Sicht der Meınungstreiheit (in: v. Munch/Kunig, GG, Bd. 1, Art. s Rdnr. 8-10; München 1992).

9 Hannah Arendt (1967), Wahrheit und Politik, in: Wahrheit und Luge in der Politik. Zwei Essays, Muinchen/Zurich 1987 (2. Aufl.). 
zunächst auf ihre Argumente eingehen, die sie für die Ausgrenzung der Wahrheitsfragen aus der freien Diskussion der politischen Öffentlichkeit geltend macht. Arendt stellt das Problem der Tatsachenfeststellung in den übergeordneten Rahmen der philosophischen Frage nach der Wahrheit und unterscheidet, Vernunftwahrheit، von ,Tatsachenwahrheitc. Sie beruft sich dabei auf Leibniz, entwickelt aber eine eigene Deutung der Begriffe. Leibniz steht im doxa-episteme-Streit eindeutig auf der Seite der Metaphysiker: nur die logische Notwendigkeit der Vernunftwahrheit gehört zur epistemc, die sinnlich vermittelte Tatsachenwahrheit wird als logisch kontingent der untergeordneten doxa zugerechnet. Arendt ordnet im Unterschied dazu sowohl die Vernunft- als auch die Tatsachenwahrhcit der Seite der episteme zu und grenzt beide von der Meinung ab. Wichtig ist die Unterscheidung zwischen Vernunft- und Tatsachenwahrheiten für sie deshalb, weil sich damit das Wirkungsfeld der Lüge cingrenzen läßr. Dic Lüge als bewußtes Leugnen der Wahrheit ist zwar bei allen wahrheitsfähigen Aussagen möglich - wirksam ist sie aber nur bei wahrheitsfähigen Aussagen, dic nicht logisch notwendig sind: den Tatsachenwahrhcitcn. Deshalb sind die Tatsachenwahrheiten im politischen Raum besonders gefährdet, und deshalb müssen sie aus dem Meinungsstreit herausgchalten werden.

Der grundlegende Unterschied zwischen Wahrheiten und Mcinungen besteht nach Arendts Darstellung in dem jewciligen Gültigkeitsanspruch und Begründungsverfahren: Tatsachenwahrheiten erhcben wie alle Wahrheiten "den Anspruch zwingender Gültigkeit", „der jede Debatte ausschließt«, weil er »durch Übereinkunft, Diskussion oder Zustimmung weder erhärtet noch erschüttert werden kann «. ${ }^{\circ} \mathrm{Mei}$ nungen können sich dagegen auf unmittelbare Gewißheit nicht stützen, sie sind das Resultat von Überlegung und bedürfen der Bcgründung, um sich im Streit der verschiedenen Ansichten und Interessen durchsetzen zu können. Während Tatsachenbehauptungen entweder wahr oder falsch sind, gelten oder nicht gelten, können Meinungen von höherer oder minderer Qualität, mehr oder weniger überzeugend sein.

Nur in Meinungsfragen - so läßt sich Arendts Position zusammenfassen - gilt das politische Gebot der Toleranz, Wahrheitsfragen hingegen erfordern absolute Antworten. Wer das politische Recht freicr Diskussion und das liberale Toleranzgebot auf Wahrheitsfragen anwenden will, betreibt Arendt zufolge cinc Umdeutung von Tatsachen in Meinungen: "Die Trennungslinie zwischen Tatsachen und Meinungen zu verwischen ist eine der Formen der Lüge."Wer sich unliebsamer Wahrheiten entledigen will, aber "nicht über die Macht verfügt, seine Fälschung öffentlich als Wahrheit zu etablieren ", weicht auf eine andere Form der Fälschung aus, indem er »erklärt, dies sei eben seine Ansicht von der Sache, für dic er dann das Recht der Mcinungsfreiheit in Anspruch nimmt ". " Der Anspruch, das Behaupten von Tatsachen stünde unter dem Schutz der Meinungsfreiheit, wird von Arendt eindcutig zurückgewiesen.

Der starke Kontrast, der hier zwischen Tatsachenwahrheiten und Meinungen gezcichnet wird, cntsteht allerdings erst durch Arendts einscitige Einordnung des Argumentationsverfahrens. Arendt erweckt den Eindruck, als seien Begründungen zum Zweck der Feststellung von Tatsachon überflüssig oder gar unmöglich, sie suggeriert eine bruchlose Kontinuität zwischon der unmittelbaren, einzelnen Wahrnchmung und der Feststellung komplexer Ercignisse. Dabei übergeht sic auch den Evidenzverlust, der entsteht, wenn Ereignisse nur noch durch Dokumente und Augenzeugen belegt, aber nicht mehr unmittelbar beobachtet werden können.

10 Arende (1967), $59 \mathrm{ff}$.

It Arendt (1967), 73. 
An Arendts Beispielsatz für eine Tatsachenwahrheit läßt sich zeigen, wie voraussetzungsvoll die Feststellung nur eines einzelnen Ereignisses ist. Die Aussage "Im August 1914 fielen deutsche Truppen in Belgien ein « ist nicht nur sprachlicher Ausdruck beobachtbarer "elementarer Daten ${ }^{12}$, wie Arendt es darstellt, sondern impliziert die Kenntnis besonderer sozialer Konventionen und Umstände, um richtig angewendet zu werden. Es ist keineswegs abwegig, die Verwendung einzelncr Begriffe wie z. B. neinfallen « in diesem Kontext durch Argumente zu stützen, etwa durch die Erläuterung, für eine Grenzüberschreitung militärischer Einheiten habe keine Rechtsgrundlage durch internationale Vereinbarungen bestanden.

Arendts Begriff von Tatsachenbehauptungen ist offenbar zu schmal: sie reduziert die Tatsachenbehauptung auf unmittelbare Wahrnehmung, und die Wahrheit von Tatsachen auf subjektive Gewißheitserlebnissc, ohne zu beachten, daß Tatsachenbehauptungen selbst keine unmittelbaren Wahrnehmungen, sondcrn Aussagen über Wahrnehmungen sind, die die Struktur begründbarer Urteile haben. Wenn eine Tatsachenbehauptung ihre Wahrheit aber nicht allein durch Beobachtung erweisen kann, sondern auf argumentative Begründung angewiesen ist, bci der sie selbst als subjektive Deutung beobachtbarer Daten zur Diskussion steht, dann liegt es nahe, Arendts Vorwurf der Transformation von Tatsachen in Meinungen umgekehrt auf ihre eigene Position anzuwenden: eine Tatsachenbehauptung, die sich mit dem Anspruch, nur Ausdruck unmittelbarer Wahrnehmung zu sein, jeder Begründung entzieht, immunisiert sich durch Transformation einer Meinung in eine Tatsache.

Um damit aber nicht ins andere Extrem des wahrheitstheoretischen Relativismus zu vcrfallen, muß man zwei verschiedene Bedeutungen von >Meinung unterscheiden: erstens gibt es Äußerungen, die deshalb keinen Wahrheitsanspruch erheben, weil sie sich auf Fragen beziehen, die selbst nicht für wahrheitsfähig gehalten werden dies können Glaubensbekundungen über die Existenz Gottes oder ein Leben nach dem Tod sein, subjektive Geschmacksurteile, Beifalls- und Mißfallensbekundungen. In diesem Sinn unterscheiden sich Meinungsäußerungen von Tatsachenbehauptungen. Zweitens gibt es Äußerungen, die als subjektive Stellungnahme zu wahrheitsfähigen Fragen auftreten, und dabei zwar einen Wahrheitsanspruch erheben, aber eben keinen "absoluten" oder "despotischen " in Arendts Sinn: sie gehen vielmehr von der eigenen Fehlbarkeit und Begründungsbedürftigkeit aus. In diesem zweiten Sinn zählen zu den Meinungsäußerungen auch Tatsachenbehauptungen.

Sowohl Arendt als auch das Bundesverfassungsgericht verbinden kurzschlüssig die Unterscheidung zwischen Äußerungen mit Wahrheitsanspruch und solchen, die keinen Wahrheitsanspruch erheben, mit der Unterscheidung zwischen subjektiven Äußerungen und objektiven Tatsachen, um dann den diskutierbaren subjektiven Meinungsstreit der nicht diskussionsfähigen objektiven Feststellung gegenüberzustellen. Statt aber Subjektivität und Begründung schlechthin als Differenzierungskriterium aufzufassen, muß zwischen verschiedenen Formen der Subjektivität und der Begründung unterschieden werden.

Eine Preisgabe des "despotischen "Wahrheitsanspruchs im Sinne Arendts bedeutet nicht, daß der Begriff der Wahrheit selbst seinen Absolutheitscharakter verliert. Gegensätzliche Aussagen über Tatsachen können nicht gleichermaßen als wahr behauptet werden. Wer einen Wahrheitsanspruch erhebt, muß im Zweifclsfall begründen können, daß seine Behauptung den Kriterien der Eindeutigkeit, Widerspruchsfreiheit und Nachprüfbarkeit entspricht. ${ }^{13}$ Dagegen kann jemand, der eine Meinung im

12 Arendt (1967), 58

${ }_{3}$ Auf die Vereinbarkeit von Wahrheitsanspruch und Pluralismus in Abgrenzung sowohl gegen relativisti- 
Sinne eines subjektiven Geschmacks-, Glaubens- oder Werturteils vertritt, sich allein darauf berufen, daß er dies eben so empfinde. Argumente können hier zwar auch das Ziel haben, andere zur Übernahme der eigenen Meinung zu motivieren, aber das Nebeneinanderbestehen verschiedener Meinungen führt nicht zu Widersprüchen und damit auch nicht notwendig zum Konflikt. Einen problematischen dritten Bereich zwischen Wahrheits- und Glaubens- bzw. Geschmacksfragen bilden Äußerungen über die moralische Richrigkeit von Handlungsregeln, die sowohl mit dem wahrheitsanalogen Anspruch auf rational begründcte Entscheidbarkeit auftreten können, als auch mit dem ermäßigten Anspruch des subjektiven Appells.

Arendts Diagnose der Umdeutung von Tatsachen in Meinungen könntc nun so rekonstruiert werden, daß Tatsachenbehauptungen sich mit dem Hinweis, sie seien lediglich Ansichtssache, einer stichhaltigen Begründung entziehen wollen. Gegenüber dieser rhetorischen Immunisierungsstrategie kann man dann darauf verwcisen, daß es sich bei Tatsachenfragen eben nicht um Fragen willkürlicher subjektiver Ansichten handelt, sondern um Fragen der Wahrheit, und daß Wahrheitsansprüche ihre Geltung auf bestimmte Standards argumentativer Begründung stützcn müssen, wenn sie überzeugen sollen. Was in dieser Lesart jedoch nicht möglich ist, ist die Abweisung des Rechts auf Meinungsfreiheit für die in Frage stehende Äußcrung. Einer Tatsachenbehauptung deshalb das Recht auf freie Äußerung streitig zu machen, weil sie die geforderten argumentativen Begründungsstandards nicht erfüllt, scheint mir ein unangemessenes Vorgehen zu sein. Aber genau solch ein Vorgehen will die Stellungnahme des Bundesverfassungsgerichts begründen.

Das Bundesverfassungsgcricht war, wie Arendt, in seiner Stellungnahme von der Unterscheidung zwischen nicht-wahrheitsfähigen Meinungen und überprüfbaren Tatsachenbehauptungen ausgegangen. Meinungen genießen »den Schutz des Grundrechts, ohne daß es darauf ankommt, ob die Äußerung begründet oder grundlos, emotional oder rational ist, als wertvoll oder wertlos, gefährlich oder harmlos eingeschätzt wird «, "Die bewußt oder erwiesen unwahre Tatsachenbehauptung " wird dagegen "nicht vom Schutz der Meinungsfrciheit umfaßt"; "Erwiesen unrichtige Tatsachenbehauptungen sind kein schützenswertes Gut «. ${ }^{14}$

Diese Auffassung wirft die Frage auf, durch welches Verfahren die Wahrheit oder Unwahrheit von Tatsachenbehauptungen erwiesen werden soll, und mit welcher Begründung unwahre Tatsachenbehauptungen von der Meinungsfreiheit per definitionem ausgeschlossen werden dürfen. Zur Klärung der Position des Bundesverfassungsgerichts muß z wischen verschiedenen Typen der freien Äußerung differenziert werden, da Artikel s des Grundgesetzes sowohl die Meinungs- und Pressefreiheit als auch die Freiheit von Kunst und Wissenschaft regclt. Die vom Bundesverfassungsgericht vertretene Einschränkung des Rechts der freien Äußerung bezicht sich nur auf dic allgemeine Meinungs- und Pressefreiheit, nicht auf die Frcihcit der Wissenschaft. Eis geht dem Bundesverfassungsgericht um die Grenzen der freien Meinungsäußerung in der politischen Öffentlichkeit, von deren Beschränkung die wissenschaftliche Auseinandersetzung als professionalisiertc Instanz zunächst unberührt bleibt. Nicht jeder Widerlegungsversuch und jede gegenteilige Äußerung einer einmal festgestellten Wahrheit soll verboten werden können. Für die Feststellung der Tatsachenwahrheit sind, so könnte man die Frage nach dem zulässigen Verfahren beantworten, die professionellen Instanzen der Wissenschaft und Rechtsprechung zuständig, die an definierte Regeln der Bcgründung gebunden sind. Der Meinungs-

sche als auch fundamentalistısche Posıtionen verweıst $\mathrm{H}$. J. Wendel in semem Beitrag: Die Intoleranz der W/ahrheit. Zur moralischen Bewertung methodologischer Strategien, in: Ethik und Sozialwissenschaften $S(1994), \mathrm{Nr} .4$.

14 I BvR $23 / 94$, S. 9 ff., 18 f́. (siehe auch Anm. 8). 
strcit in der politischen Öffentlichkeit ist solchen Regcln nicht unterworfen, muß sich aber den Ergebnissen der Wahrheits-Experten unterordnen.

Das mögliche Verbot unwissenschaftlicher und erwiesen unwahrer Behauptung kommt insofern dem Versuch gleich, die Einhaltung wissenschaftlicher Regeln der Wahrheitsfeststellung auf dem Weg der Rechtsprechung zu sichern. Dabei werden allerdings bedingte Vorschriften, die nur befolgt werden müssen, wenn ein bestimmtes Ziel - Wissenschaftlichkeit - erreicht werden soll, und deren Mißachtung durch die "Strafe* ihres Mißcrfolgs sanktioniert ist, zu unbedingt verpflichtenden Geboten, deren Einhaltung durch festgesetzte Sanktionen gesichert werden.

Nehmen wir z. B. an, es werde dic Behauptung, daß bcstimmte Bodenzeichnungen im Wüstensand an der Küste Perus von außerirdischen Intelligenzen als Landebahnen für Raumschiffe angelegt worden seien, auf öffentlichen Versammlungen verbreitet, obwohl sie wissenschaftlich hinreichend widerlegt worden sei. Nichts würde dazu berechtigen, eine solche Theorie der Außerirdischen zusätzlich zum Nachweis ihrer wissenschaftlichen Unhaltbarkeit auch noch gerichtlich zu verbieten. Vielmehr wäre zu befürchten, daß ein solches Verbot dieser Theorie unangemessen viel Aufmerksamkeit verschaffen und ihre Vertreter gerade zu Märtyrern im Dienste der Wahrheit stilisieren könnte. Der Schluß liegt nahe, daß auch das Verbot der Leugnung des Holocaust in dieser Hinsicht grundsätzlich nicht besser begründet ist.

Die vom Bundesverfassungsgericht unterstellte Kompetenzverteilung zwischen Experten und Öffentlichkeit widerspricht der liberalen Auffassung des Grundrechts auf Meinungsfrciheit, die das öffentliche Interesse am freien Meinungsstreit betont; das Bundesverfassungsgericht scheint dagegen das Recht auf freie Meinungsäußerung nur als subjektives Gut zu betrachten. Die liberale Position in der Tradition John Stuart Mills geht davon aus, daß es im öffentlichen Intercsse licgt, die Freihcit der Mcinungsäußerung nicht zu behindern, weil nur auf diesem Weg Wahrheit gesichert werden kann.' Im Unterschicd zum Bundesverfassungsgericht geht diese Position von ciner prinzipiellen Unabgeschlossenheit des Prozesses der Wahrheitsfindung aus. Das Kriterium erwiesener Wahrheit oder Unwahrheit kann daher nicht zur Begrenzung der Meinungsfreihcit dienen.

Dagegen kann natürlich eingewendet werden, daß im juristischen Alltag ständig über Tatsachenfragen entschieden werden muß, und der Verweis auf die grundsätzliche Fchlbarkeit unscres Wissens hier zu vollständiger Handlungsunfähigkeit führen würde. Dieser schcinbarc Gegensatz löst sich aber auf, wenn zwei Ebenen beim Verfahren der Wahrheitsfindung unterschieden werden: zum einen die rechtich verbindliche Entschcidung über praktisch relevante Tatsachenfragen, dic von autorisierten Instanzen wie sachverständigen Gutachtern und Gerichten getroffen wird, zum andercn der zeitlich und räumlich unbegrenzte Diskurs über Wahrheitsfragen, an dem jeder beteiligt ist, der mit sciner Aussage öffentlich einen Wahrheitsanspruch erhebt. Dic Schlichtung aktueller Konflikte und Interessenkollisionen bedarf der Verbindlichkeit juristischer Entscheidungen. Die historische Wahrheit aber, die sich in den Überzeugungen der Menschen durchsetzen wird, kann damit nicht festgelegt wcrden. ${ }^{16}$

is \#nsere gesıchertesten Úberzeugungen haben keıne verlaßlıchere Schutzwache als eine standige Einladung an die ganze Welt, sie als unbegrundet zu erwersen. (...) Eine Behauptung gesıchert nennen, solange es jemanden gibt, der ihre Sicherhett abstretten wurde, wenn es ihm erlaubt ware - der diese Erlaubnis aber nicht erhalt - heißt annehmen, daß wir und die mit uns Gleichgesinnten Richter uber die GewrßSheıt sind und noch dazu richten, ohne die Gegenseite anzuhoren. * John Stuart Mill (1859): On Liberty. Ubersetzt von B. Lemke, Stuttgart 1988, S. 31 f.

16 Eine ahnlıche Unterscheidung legt auch Kant bet seiner *Beantwortung der Frage: Was ist Aufklarung? zugrunde, wenn er den offentlichen Gebrauch der Vernunft, sden jemand als Gelehrter von ihr vor dem ganzen Publikum der Leserwelt macht $\propto$, vom Privatgebrauch abhebt, den jemand «n einem gewissen ihm 
Zusammengefaßt hat die Position des Bundesverfassungsgerichts gravierende Schwächen: Hier wird nicht nur die Bedeutung des öffentlichen Meinungsstreits für das Verfahren der Wahrheitsfindung unterschätzt, sondern es wird außerdem der theoretische Diskurs über Wahrheitsfragen nicht ausreichend vom praktischen Verfahren verbindlicher Tatsachenfeststeilung unterschieden. Der Versuch, einc grundsätzliche Einschränkung des Rechts der freien Äußerung bereits auf der bcgrifflichen Ebene vorzunehmen, und es nur für $>$ Meinungen im Sinne bewertender Äußerungen ohne Wahrheitsanspruch gelten zu lassen, ist mit der liberalen Auffassung des Grundrechts der Meinungsfreiheit unvereinbar. Grundsätzlich muß auch in der Öffentlichkeit über Wahrheitsansprüche und übcr die juristisch oder wissenschaftlich als wahr erwiesenen Tatsachenbehauptungen gestritten werden dürfen. Der Vcrweis auf professionelle Instanzen der Wahrheitsfeststellung kann die Befassung der Öfentlichkeit mit Fragen der Wahrheit nicht ersetzen, er birgt vielmehr die Gefahr der Dogmatisierung und Immunisierung von "Herrschaftswissen «. Die staatliche Berufung auf endgültige Wahrhciten, unabhängig vom Fürwahrhalten der Öffentlichkeit, ist philosophisch unhaltbar und politisch gefäbrlich - auch wenn es sich um historische Ereignisse von höchster Bedeutung wie im Fall des Holocaust handelt.

\section{II}

Wenn das Grundrecht auf freie Meinungsäußerung nicht schon durch die epistemische Unterscheidung der Meinungs- von den Wahrheitsfragen begrenzt werden kann, so besteht seine Einschränkung doch unbestritten im möglichen Konflikt mit anderen Rechten. In Art. s GG wird dieses Recht - im Unterschied zur Freiheit der Kunst und Wissenschaft, die nur der Treue zur Verfassung unterliegen - ausdrücklich in die Schranken der Vorschriften der allgemeinen Gesetze, der gesetzlichen Bestimmungen zum Schutze der Jugend und des Rechtes der persönlichen Ehre verwiesen. Dieser Aspekt einer Abwägung zwischen konfligierenden Rechten begründet auch die begrenzte Vergleichbarkeit des angeführten Beispiels der peruanischen Bodenzeichnungen mit dem Fall der Holocaust-Lcugnung: Hier steht eben nicht allein die Leugnung der historischen Tatsache des Holocaust auf dem Spiel, sondern auch das Schutzrecht von Personen, deren Identität mit der Tatsache des Holocaust verknüpft ist. Genauer gesagt geht es hier um die Abwägung zwischen dem Freiheitsrecht der ungehinderten Meinungsäußerung auf der einen Seite, dem Schutzrecht der Menschen würde nach Art. I GG (bzw. dem früheren $\$ I $30 \mathrm{OtGB}$ ) und der persönlichen Ehre nach den $\$ \$ 18$ sff. BGB auf der anderen Seite.

Eine solche Abwägung nimmt das Bundesverfassungsgericht in seinem Urteil abschließend vor und kommt zu dem Schluß, daß die Meinungsfreiheit in diesem konkreten Fall hinter dem Persönlichkeitsschutz zurücktreten müsse. Die "Leugnung der rassisch motivierten Vernichtung der jüdischen Bevölkerung im Dritten Reich « stche in einem direkten Zusammenhang mit »dem Angriff auf den Achtungs-

anvertrauten bürgerlichen Posten, oder Amte, von seiner Vernunft machen darf $\alpha$ : „der offentliche Gebrauch seiner Vernunft muß jederzeit frei sein, und der allein kann Aufk larung unter Menschen zu Stande bringen*. (Immanuel Kant, Beantworung der Frage: Was ist Aufklarung?, A ${ }_{484}, 485$. Zicier nach: Schriften zur Anthropologic, Geschichtsphilosophie, Politik und Padagogik r, herausgegeben von W. Weischedel, Frankfurt/M. 1977). Naturlich laßt sich hier einwenden, dic crwiesen falsche Tatsachenbehauptung sei gerade kein Fall von sVernunftgebrauch «, sondern ein irrationaler Akt - doch das hieße eben, sich zum Richter über die Gewißheit machen bzw. die Ergebnisse des Aufklarungsprozesses vorwegnehmen zu wollen. 
anspruch und die Menschenwürde der heute lebenden Juden “. ${ }^{17}$ Das Bundesverfassungsgericht vertritt damit die Auffassung, daß die Leugnung des Holocaust nicht nur einen Angriff auf die persönliche Ehre, sondern auch auf die Menschenwürde darstelle. Der Bundesgerichtshof hingegen hattc für den Straftatbestand der Volksverhetzung, der bislang ja an das Kriterium eines Angriffs auf die Menschenwürde gebunden war, dic Bedingung geknüpft, ein solcher Angriff liege nur dann vor, wenn nachgewiesen werden könne, "daß der angegriffenen Person ihr Lebensrecht als gleichwertige Persönlichkeit in der staatlichen Gemeinschaft abgesprochen und sie als minderwertiges Wesen behandelt wird $«{ }^{18}$ Gerade diese Forderung hatte zu großer öffentlicher Empörung geführt und zur Änderung des $\$$ I 30 StGB erheblich beigetragen.

Dabei geht es um die Frage, ob das Bestreiten eines historischen Verbrechens einen direkten Angriff auf die Würde der Opfer darstellt; anders formuliert: Ob das Bestreiten historischer Tatsachen, die Teil der identitätskonstitutiven Gewißheiten bestimmter Personen sind, eine rechtlich strafbare Mißachtung ihrer Würde darstellt. Strittig ist insbesondere, wie hier der Begriff der Würde im Unterschied zur persönlichen Ehre auszulegen ist, und wodurch die Würde bedroht wcrden kann.

Durch das Bestreiten historischer Tatsachen, die Teil der identitätskonstituierenden Gewißheiten einer Person sind, wird auch die Anerkennung der Identität dieser Person verweigert. Eindeutig sind in diesem Sinn Fälle, in denen ein direkter Bezug zwischen der Tatsachenbehauptung und der Person besteht, wenn es sich also z. B. um die Behauptung handelt, eine bestimmte Person habe eine Handlung begangen, die eindeutig moralisch zu verurteilen ist, wie dies bei der Unterstellung einer Lüge geschieht. Solche Tatsachenbehauptungen zielen direkt auf die persönliche Ehre einer Person. Nicht ganz so deutlich liegt der Fall bei Behauptungen, die anderen Personen nur indirekt moralisch verwerfliche Absichten oder herabsetzende Eigenschaften unterstellen. Hier hängt die Beurteilung, ob eine solche Äußerung eine Beleidigung darstellt, auch davon ab, wie offensichtlich der beleidigende Zusammenhang ist, ob also eine Absicht oder mindestens die billigende Inkaufnahme der Ehrverletzung unterstellt werden muß.

Tatsachenbehauptungen (oder die Leugnung von Tatsachen) die auf diese Weise im Zusammenhang mit identitätskonstituierenden Gewißheiten stehen, sind als Beleidigung, üble Nachrede, Verleumdung und Verunglimpfung nach den $\$ \$ 18 \mathrm{sf}$. StGB strafbar. In bezug auf den juristischen Umgang mit der Leugnung des Holocaust und der Behauptung der "Auschwitzlüge « kann es hier allenfalls Differenzen in der Einschätzung des konkreten Falles geben, es besteht aber kein grundsätzlicher Regelungsbedarf, der eine vermeintliche Gesetzeslücke schließen müßte. Insofern die Leugnung des Holocaust und das Behaupten der "Auschwitzlüge « eine Beleidigung, Verleumdung und Verunglimpfung der Opfer darstellt, ist sie nach geltendem Recht eindeutig strafbar.

Grundsätzliche Differenzen bestehen allerdings in der Frage, ob die Leugnung des Holocaust neben diesem Angriff auf die persönlichc Ehre auch ein Angriff auf die Menschenwürde ist. Stellt die Mißachtung des konkreten Selbstverständnisses einer Person einen Angriff auf ihre Menschenwürde dar, oder besteht die Menschenwürde allein im Anspruch auf Anerkennung der allgemeinen Subjektqualitäten der personalen Eigenverantwortichkeit und Gleichwertigkeit? Gerade weil der Begriff der Menschenwürde einen allgemeinen Achtungsanspruch begründet, der unabhängig von den konkreten persönlichen Eigenschaften und Lebensumständen besteht, kann

171 BvR $23 / 94$, S. 17 .

18 NJW 1994, 1421 . 
er nicht in Fällen Anwendung findon, in denen es um dic Anerkennung konkreter identitätskonstitutiver Gewißheiten geht, die über die allgemeinen Subjektqualitäten hinausreichen. Insofern stimme ich der Beurteilung des Bundesgerichtshofs zu, daß das bloße Bestreiten des Holocaust selbst keinen Angriff auf die Menschenwürde darstellt. Ein solcher Angriff auf die Menschenwürde kann jedoch in der aufhetzenden Agitation liegen, die sich gegen bestimmte Personen richtet und deren Recht auf Leben und Unversehrtheit direkt oder indirekt bestreitet. ${ }^{19}$

Nun muß man sagen, daß diese Diskussion inzwischen nicht mehr von praktischer Bedeutung ist, weil in der Neufassung des $\$$ r 30 StGB nicht nur die Leugnung des Holocaust ausdrücklich in den Straftatbestand der Volksverhetzung aufgenommen wurde, sondern darüber hinaus auch auf den Nachweis eines Angriffs auf die Menschen würde verzichtet wird. Es handelt sich damit um den nicht zu unterschätzenden Sonderfall eines Gesetzes, der das Lcugnen ciner historischen Tatsache nur unter Hinweis auf die mögliche Störung des öffentlichen Friedens unter Strafe stellt. Die Entscheidung, das Strafgesetz in dieser Weise zu novellieren, halte ich in juristischer Hinsicht aus zwei Gründen für falsch. Schon die ausdrückliche Anführung der Leugnung des Holocaust als Straftatbestand ist falsch, weil nicht die Leugnung der historischen Tatsache, sonder der damit verbundene verletzende Angriff auf andere die Straftat darstellt. Den gleichzeitigen Verzicht auf das Kriterium eines Angriffs auf die Mcnschenwürde für den Straftatbestand der Volksverhetzung aber halte ich für fatal. Die Billigung und Verharmlosung von Völkermord ist ohne Angriff auf die Menschenwürde nicht denkbar und kann insofern vielleicht auf die ausdrückliche Nennung dicses Kriteriums verzichten, bei der Leugnung ist dieser Zusammenhang jedoch nicht in gleicher Weise zwingend. Die verbliebene einzige Bedingung einer möglichen Störung des öffentlichen Friedens ist meiner Ansicht nach im Hinblick auf die Abwägung 2 wischen konfligierenden Rechten kcin hinreichendes Kriterium für die Einschränkung der Mcinungsfrciheit.

Meines Erachtens läßt sich diese Form der Neufassung des $\$ 130 \mathrm{StGB}$ ohne Bezugnahme auf die besonderc psychologische Bedeutung des Holocaust in der politischen Öffentlichkeit und die gegenwärtige politische Situation in Deutschland nicht erklären. Was der Wortlaut des Gesetzes sclbst nicht mehr erkennen läßt, kann nur noch durch die Kenntnis der aktuellen politischen Lage nachvollzogen werden: die paradoxe Absicht nämlich, daß gerade durch Weglassung des Kriteriums eines nachweisbaren Angriffs auf die Menschenwürde dem Zusammenhang zwischen der Strafbarkeit der Holocaust-Leugnung und den Schutzrechten anderer Rechnung getragen werden soll. Die politische Entscheidung, in der Neufassung des $\$$ I 30 SiGB den Straftatbestand eincs Angriffs auf die Menschenwürde wegzulassen, hat vermutlich ja gerade niche den Grund, daß ein solcher Angriff hier als irrelevant betrachtet wird, sondern im Gegenteil, daß unabhängig von den konkreten Umständen unterstellt wird, daß jeder Versuch, die historische Tatsache des Holocaust zu leugnen, per se einen Angriff auf die Menschenwürde darstellt. Und daß aus diesem Grund der bisherige Ermessensspielraum der Richter, denen nach einigen politisch stark um-

19 Die verkurzte Wiedergabe des entsprechenden BGH-Urteıls (siehe Anm. 4) in den Medien hat nicht unerheblich dazu beigetragen, daß der emporende Eindruck entstand, der Bundesgerichtshof behaupte in seinem Urteil, daß der Angeklagte auch gar keınen Angriff auf die Menschen wurde beabsıchtige - und sei damit sauf die Pose des nur der Wahrheit verpflichteten Forschers herengefallen*. Der Bundesgerichtshof hatte jedoch nur festgestellt, daß die falsche Tatsachenbehauptung allem fur das Delike der Volksverhetzung nicht ausreiche, und daß eine solche Verurteilung sich gegebenenfalls auf Begleıtumstande wie wbesondere Gesten des Angeklagten, die Betonung einzelner Passagen durch Stimmlage oder Lautstarke sowie kommentuerende Bemerkungen a stutzen musse (Helmut Kerschner, Die Luge bleibt ene Luge, Suddeutsche Zeitung 23.6.94. Eme ahnliche Einschatzung außert auch G. Bertam: Entrustungssturme im Medienzetalter - der BGH und die "Auschwutzlugen, NJW 1994, 2002-2004). 
strittenen Äußerungen eine angemessene Beurteilung konkreter Fälle nicht mehr

ohne weiteres zugetraut wird, drastisch eingeschränkt werden kann und muß.

Dic Ungehcucrlichkcit des moralischen Verbrechens, das mit dem Faktum der systematischen und massenhaften Ermordung anderer Menschen durch die Deutschen verbunden ist, läßt es einerseits als undenkbar erscheinen, daß das Bestreiten dieses Faktums in der unschuldigen Absicht bloßer Wahrheitsfindung geschieht. Auf der anderen Seite ist die moralische Schuld gegenüber den Opfern so unermeßlich groß, daß jede weitere Verletzung der Schutzrechte dieser Personen in ganz besonderer Weise als unerträglich empfunden werden muß. Daher erscheint dann selbst die Forderung, eine solche Verletzung der Fhre und der Menschenwürde dieser Opfer durch das Leugnen ihrer Qualen müsse im einzelnen nachgewiesen werden, als unverhältnismäßige Zumutung.

Der Umgang mit der Holocaust-Leugnung, der in dieser Gesetzesnovellierung zum Ausdruck kommt, ist - trotz solcher gut gemeinter Motive - jedoch auch in politischer Hinsicht nicht zweckmäßig. Er dient der Aufrechterhaltung eines Tabus auf eine Weise, die langfristig gesehen eher schaden als nützen wird. Mit zunehmendem zeitlichen Abstand von den historischen Verbrechen der Deutschen in der Zeit des Nationalsozialismus wird dic Evidenz dieser Tatsachen im Bcwußtsein der Öffentlichkeit schwinden.* Irgendwann wird es keine lebenden Zeitzeugen, weder unter den Opfern noch unter den Tätern, mehr geben. Damit schwindet auch die Evidenz der moralischen Dimension der Leugnung des Holocaust. Die Ähnlichkeit mit dem Beispiel der peruanischen Bodenzeichnungen wird im Empfinden der dann nachfolgenden Generationen immer größcr werden: sie werden mit einem Kapitel der Geschichte konfrontiert, dessen Infragestellung strafbar ist. Unter Bcrücksichtigung des Umstands, daß der Holocaust für junge Menschen dann zunehmend den Status eincr Erzählung annehmen könnte, die geglaubt werden muß, ist eine solche Tabuisierung möglicher Zweifel an den Tatsachen politisch schädlich. Selbstverständlich wird es auch in dreißig Jahren noch die Dokumente geben, die die historische Tatsache des Holocaust zweifelsfrei belegen. Gerade deshalb aber ist es nicht sinnvoll, die. Tatsache des Holocaust mit einem Tabu zu belegen, das ihre Infragestellung sanktioniert. Anders ist es bei der moralischen Bewertung des Holocaust: nicht das Tabu gegenüber dem Verbrechen als ungeheuerlichem Verbrechen soll gebrochen werden, sondern gegenüber dem Verbrechen als einem nachweisbaren historischen Faktum. Ein Gesetz, das die Tatsache des Holocaust tabuisiert, dient - wie ungewollt auch inmer - ihrer Angreifbarkeit als ideologisch motiviertem Mythos. Nicht Rechtsvorschriften können die historischen Tatsachen vor dem Vergessen, Verdrängen und Verfälschen schürzen, sondern die öffentliche Erinnerung und Thematisierung dessen, was geschehen ist.

Die Konfrontation mit der Behauptung einer »Auschwitzlüge" stellt uns nicht nur vor die epistemologische. Frage nach dem Verfahren der Wahrheitsbegründung und die moralische Frage nach der Anerkennung persönlicher Idencitäten, sondern drittens auch vor die politische Frage unserer nationalen Identität. Der Holocaust als historisch beispiellosc systematischc Massenvernichtung, die sich nicht auf die Täterschaft einiger weniger reduzieren läßr, hat allen Deutschen eine besondere Verpflichtung hinterlassen, sich dafür einzusctzen, daß Ähnliches nicht wieder geschieht. Die historische Tarsache des Holocaust ist daher nicht nur mit der Anerkennung der Identität der Opfer und ihrer Hinterbliebenen eng verbunden, sondern auch mit der

S Anm.der Redaktion:

Die Leugnung ist in $\$ 1$ zo Abs. 3 StGB neue Fassung (vom 1. 12. 1994) strafbar, wenn sie sgeeignet ist, den offentlichen Frieden zu storen-. Abs. 4 sieht etwa bei swissenschafelichen* Debatten vor, von Strafe abzuschen bzw. ein Strafverfahren einzustellen. 
Anerkennung der Identität der Täter als Täter, und ihrer Nachkommen als einer Nation, die eine kollektive Verantwortung trägt. Diesen politischen Konsens über die Lehren aus der Vergangenheit wird auch ein auf Lückenlosigkeit bedachtes Recht nicht ersetzen können. Es kann im Gegenteil eher dazu führen, die notwendige politische Auseinandersetzung über unsere Identität und Verantwortung durch überzogenc Regelungen einzufrieren.

Die im Titel gestellte Frage nach der Reichweite der Meinungsfreiheit will ich abschließend daher so beantworten: Die Leugnung des Holocaust stellt den Härtefall für das liberale Verständnis der Meinungsfreiheit dar, der uns zu Bewußtsein bringt, daß es auf die Frage nach Wahrheit auch dann keine endgültigen Antworten gibt, wenn wir uns dies aus moralischen oder politischen Gründen wünschen. Tatsachenwahrheiten mögen noch so gut dokumentiert und begründet sein - nichts kann sie davor schürzen, bezweifelt zu werden, und nichts gibt uns das Recht, sie als Tatsachenwahrheiten von vornherein der öffentlichen Diskussion zu entzichen. Das Grundrecht der freien Meinungsäußerung findet seine Grenze in den Rechten anderer Personen, die vor Beleidigung und Verletzung geschützt werden müssen, es darf aber nicht durch ein juristisch sanktioniertes Delikt der ,Lüge،, ,Unwahrhaftigkeit، oder ,Unbelehrbarkeit beschränkt werden, durch das der öffentliche Konsens über Tatsachenwahrheiten jedem Zweifel enthoben werden soll. An die Stelle letzter Gewißheiten kann hier nur eine politische Übereinkunft treten, die festlegt, wer wir sein und wofür wir uns einsetzen wollen. 